\title{
Investigating the effect of ghrelin on improving memory and learning ability in animals: A systematic review
}

\author{
Mohaya Farzin $1,2,{ }^{*}$ Parastoo Jafarzade¹, Niloofar Faraji², Seyedeh Maral Mousavi², Mostafa Yousefi ${ }^{3}$ \\ ${ }^{1}$ Department of Physiology, School of Medicine, Guilan University of Medical Sciences, Rasht, Iran \\ ${ }^{2}$ Razi Clinical Research Development Unit, Razi Hospital, Guilan University of Medical Sciences, Rasht, Iran \\ 3Department of Biology, Faculty of Sciences, University of Guilan, Rasht, Iran
}

\begin{abstract}
Ghrelin does an important role in neurological functions such as memory, learning, and cognition as well. There is some evidence that ghrelin can improve memory. This study aimed to evaluate the effects of ghrelin on rat memory. A systematic search was conducted to identify papers published by Iranian authors in the Web of Science, PubMed, Scopus, Embase, and Google Scholar electronic databases from January 2010 to December 2019 with the PRISMA statement. Totally, 547 animals were listed in our systematic review. All of the animals were male Wistar rats with a mean weight of $250 \pm 280 \mathrm{~g}$. The Morris Water Behavior behavioral test and the passive avoidance task were performed to evaluate memory. Truly, injection of ghrelin appears to enhance the compactness of the synapse dendritic spines in the hypothalamus. These findings indicate that ghrelin has a prospective role to recover learning and memory ability in animal models, suggesting it as a candidate therapy for memory.
\end{abstract}

Keywords: Ghrelin, Memory, Depression, Memory disorders

\section{Introduction}

Ghrelin is a 28 -amino acid orexigenic peptide that was identified as an endogenous ligand for the growth hormone secretagogue receptor (GHSR)-1a, performing a significant role in nutrition intake, control of obesity, and plays an important role in homeostasis [1]. Based on the findings, it showed that the hippocampus plays an important role in cognitive function and is a vital structure for memory [2]. The presented in different brain tissues for example the hypothalamus and pituitary, ghrelin control brain purposes by entering or highly mainly as GHS-R1a mRNA in hippocampal neurons [3]. Studies have shown that ghrelin is present in hypothalamic neurons, which are located next to the third ventricle between the abdominal, dorsal, ventricular, and

\footnotetext{
${ }^{*}$ Corresponding author:

Mohaya Farzin, Ph.D

Razi Clinical Research Development Unit, Razi Hospital,

Guilan University of Medical Sciences, Rasht, Iran

Tel/Fax: +98 1333542460

Email: mf99155@yahoo.com

https://orcid.org/oooo-0003-4739-2454
}

Received: February, 28, 2021

Accepted: April, 06, 2021 arched nuclei [4]. Notably, ghrelin neurons are located in the hypothalamus and cortex, also the ghrelin neuron fibers in the hypothalamus are straightly thrust to the dorsal vagal complex (DVC) [5]. The above-mentioned ghrelin-induced synaptic growth causes better spatial learning and memory while meddling in ghrelin gene expression diminished the spine synapses density and cholinergic fibers in the hippocampal CA1 which caused reasoning and memory performance to lessen [6]. Moreover, intra amygdaloid ghrelin management enhances learning and memory procedures in aversive circumstances in male rats, as studied in the Morris water maze (MWM) patterns [6].

Intra-hippocampal injection increases long-term memory [7]. It suggests a modulatory performance of 
ghrelin in memory acquisition/consolidation over processes that could contain the promotion of synaptic plasticity and concentration of hippocampal cholinergic fibers [8]. The particular role of ghrelin in depression and anxiety is ambiguous by now, as it has shown, either advanced and lighten depressive and anxiety-behavior in animal studies are with an overweight of indication proposing antidepressant special effects. But a few human investigations demonstrate an interruption of the ghrelin system in a patient with dementia [9]. This study aims to provide a concise yet comprehensive overview of ghrelin's role in memory. Ghrelin consistently showed protective and memory-enhancing effects, as well as reducing psychopathology in animal models of dementia. Some human investigations demonstrate an interruption of the ghrelin system in a patient with dementia.

\section{Materials and Methods}

\subsection{Search strategies}

A systematic search from January 2010 to December 2019 on the Web of Science, Google Scholar, Scopus, PubMed, and Embase electronic databases was carried out. The Guidelines used for this literature review were from the preferred reporting items for systematic reviews and meta-analyses (PRISMA). The search was conducted by two reviewers who independently screened the databases through the following terms: "Ghrelin" AND "Alzheimer's disease" OR "Memory corruption" OR "Improve memory" OR "Memory disorder" OR "Cognition disorders" OR "Neurodegenerative diseases" AND Iran. Any discrepancies were resolved by consensus with a third reviewer. Studies were included without language restrictions.

\subsection{Inclusion criteria}

The following articles are included in the study (1) Ghrelin was used as a treatment, (2) The tests used in the articles include the Maurice Water Table and the Shuttle Box, (3) All studies investigating ghrelin in animals.

\subsection{Exclusion criteria}

Articles were excluded if the following standards were encountered: (1) Duplicate publications, (2)
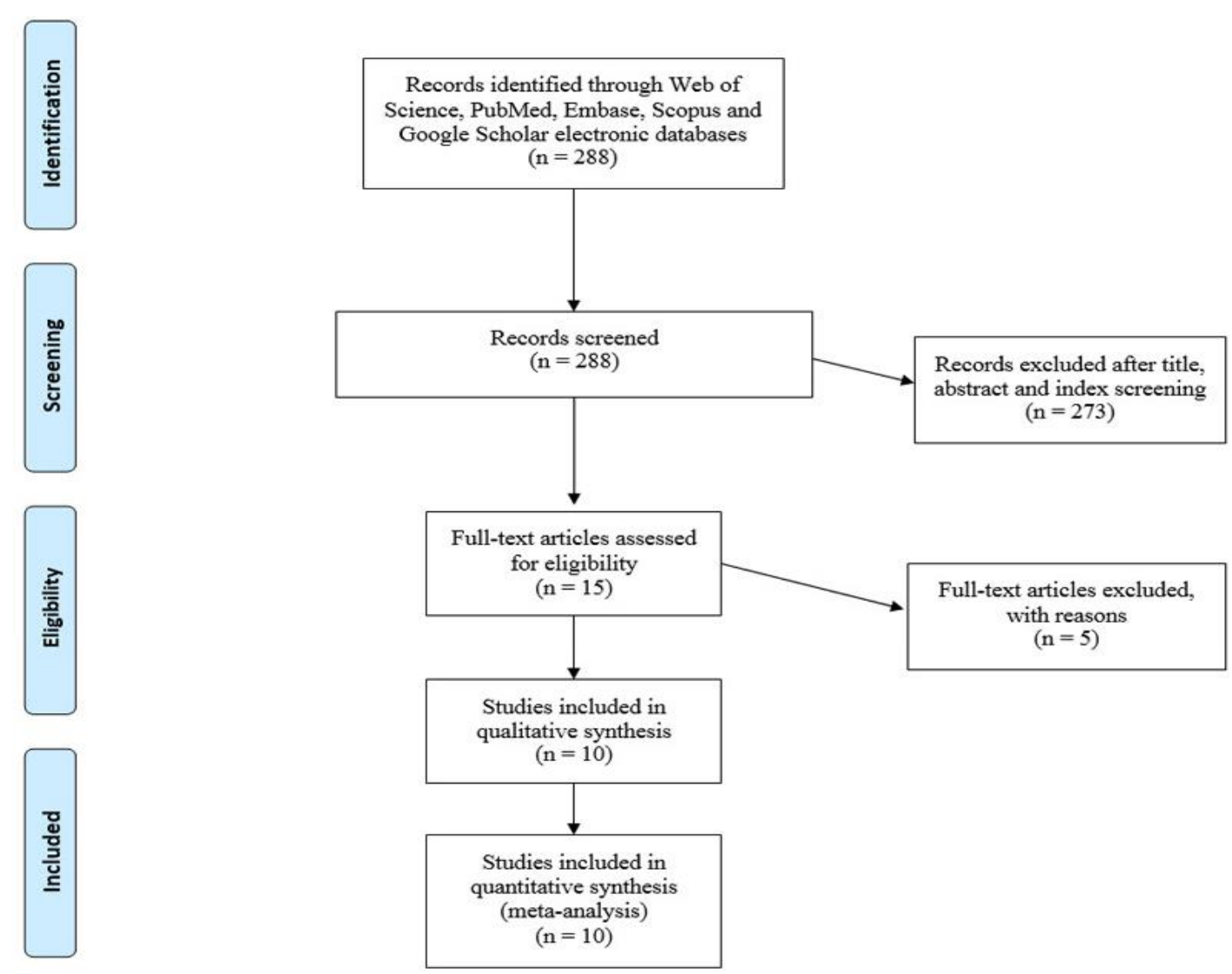

Figure. 1 PRISMA flow diagram showing a selection of articles for review 
articles that compared diverse techniques, (3) Studies with a non-control group, (4) Intraperitoneal ghrelin injection in mice.

\subsection{Data extraction}

The following details were extracted for each of the included studies: the first author's name, publication date, the study location, sample size, animal details, treatments, and outcome.

\section{Results}

Ghrelin increases memory and improves map memory performance by becoming longer and richer. Ghrelin seems to have a beneficial effect on memory disorders in animals. This will make the memory have better quality. Finally, it appears to affect synaptic flexibility in areas involved in memory and has been shown to improve memory capacity in rodents. A total of 15 potential articles were identified. After checking duplicates, reviewing titles, abstracts, and full text, 10 eligible articles were included, which detailed characteristics of them are specified in Table 1. The flow diagram outlining the searching procedures is presented in Figure 1.

Over levels of screening, 547 animals were listed in our systematic review. All of the animals were male Wistar rats. The weight of all the animals was over $250 \pm 280$. The Morris Water Behavior behavioral test and the passive avoidance task were performed to evaluate memory. Truly, injection of ghrelin appears to enhance the compactness of the synapse dendritic spines in the hypothalamus. According to both types of research by Babri, et al. ghrelin can increase memory level [10]. Also, other studies by the simultaneous use of narcotics and ghrelin indicated that ghrelin can improve memory level $[11,12]$. Using the shuttle box and ghrelin block, Zahiri et al. and Beheshti, et al. showed that the level of memory increased by ghrelin [2]. Moreover, Eslami, et al. by use of ghrelin blocking demonstrated that it can enhance memory level [6].

\section{Discussion}

This study highlights the conclusion of ghrelin on ameliorating learning and memory aptitude. To our information, this study is a systematic review that discovers the efficacy of ghrelin on enhancing memory and learning in animal trials by the results of the Morris water maze test and shuttle box as the result measures in Iran. It recommends that ghrelin has a possible role in enhancing learning and memory impairment in animal models.

Ghrelin appears to use a neuroprotective effect in various models over similar signaling pathways. Subsequently, ghrelin could be used to improve existing symptoms in multiple neurodegenerative and also neurological disorders as well as to make disease development slow. In the CA1 region of the hippocampus, ghrelin developed spatial memory and about the ghrelin secretion from the stomach through fasting, it can be theorized that ghrelin may have an ameliorating impact on learning and memory which exposed the enhancing the effect of higher doses of ghrelin on spatial memory in the PTZ-treated rats [10]. These findings indicate that the GHS-R1a blocking in the rat's brain using a selective antagonist impairs memory encoding and emphasis the idea of the signaling of endogenous ghrelin in the rat's brain is vital for memory attainment and merging [2]. A longterm schedule of ghrelin therapy can have a possible therapeutic value for impaired cognitive function and ghrelin treatment might improve spatial memory and reduce the apoptotic cell number in the hippocampus of methamphetamine-treated animals [6, 13]. Advantageous impacts of ghrelin are mediated fairly by the N-Methyl-D-aspartic acid (NMDA )receptor signaling and ghrelin in the amygdale dependent learning [14, 15]. Ghrelin in the dorsal hippocampal network avoids disrupting the influence of morphine in avoidance memory. Furthermore, ghrelin empowers the nicotine improving impact on morphine-induced impairment and also the hippocampal nicotinic receptors which are involved in this procedure [12]. Infusion of ghrelin into the lateral ventricle was identified to behave this potency to preventing morphine-induced memory impairment loss. The median septal area appears to mediate the ghrelin effects on morphine-induced amnesia [16]. Based on the co-administration of ghrelin and compound Chighlighted the reductive effect of ghrelin on memory, and it could be considered that this presentation is at least in part, mediated via the AMPactivated protein kinase (AMPK)pathway [17].

As a limitation, these data must be interpreted with caution because our population was not large enough and we only evaluated studies conducted in a limited area. Further studies with larger numbers of articles are needed to confirm our results. 
Table 1. Characteristics of studies included in the meta-analysis

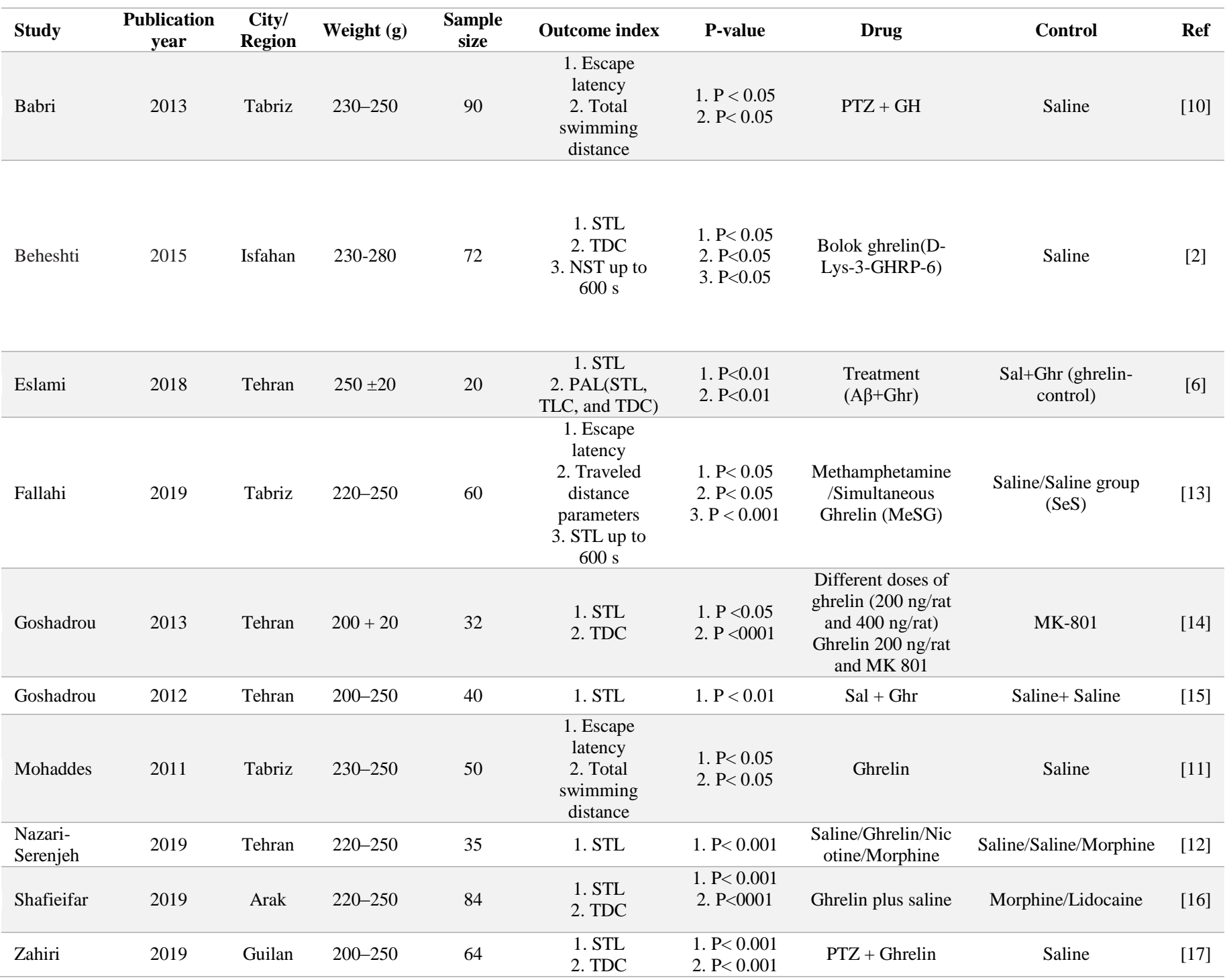

Abbreviations: MVM: Morris water maze; GH: Ghrelin; PTZ: Pentylenetetrazol; AMPK: Adenosine Monophosphate Kinase; PAL: Passive avoidance learning; STL: Step-through latency; TDC: Time spent in the dark compartment; NST: Step-through into the dark compartment; GHRP: Growth hormone-releasing peptide

In an animal model, ghrelin has a significant role to improve memory ability and learning. But the most effective stimulating (intraventricular injection of ghrelin or systemic) is still imprecise. The results of this review have shown that the effect of ghrelin improves acquisition, consolidation, and retrieval of passive avoidance memory and could reduce the time of escape latency, decrease the frequency of crossplatform.

\section{Author Contributions}

All authors contributed equally to this manuscript and approved the final version of the manuscripts.

\section{Conflict of Interests}

The authors declare that they have no conflicts of interest.

\section{Ethical declarations \\ Not applicable.}

\section{Financial support}




\section{Not applicable.}

\section{References}

1. Fabel K, Kempermann G. Physical activity and the regulation of neurogenesis in the adult and aging brain. Neuromolecular Med. 2008; $10(2): 59-66$.

2. Beheshti S, Shahrokhi S. Blocking the ghrelin receptor type 1a in the rat brain impairs memory encoding. Neuropeptides. 2015; 52:97-102.

3. Cong WN, Golden E, Pantaleo N, White CM, MaudsleyS, Martin B. Ghrelin receptor signaling: a promising therapeutic target for metabolic syndrome and cognitive dysfunction. CNS Neurol Disord Drug Targets. 2010; 9(5):557-63.

4. Cowley MA, Smith RG, Diano S, Tschöp M, Pronchuk N, Grove $\mathrm{KL}$, et al. The distribution and mechanism of action of ghrelin in the CNS demonstrates a novel hypothalamic circuit regulating energy homeostasis. Neuron. 2003; 37(4):649-61.

5. Hwang S, Moon M, Kim S, Hwang L, Ahn KJ, Park S. Neuroprotective effect of ghrelin is associated with decreased expression of prostate apoptosis response-4. Endocr J. 2009; 56(4):609-17.

6. Eslami M, Sadeghi B, Goshadrou F. Chronic ghrelin administration restores hippocampal long-term potentiation and ameliorates memory impairment in rat model of Alzheimer's disease. Hippocampus. 2018; 28(10):724-34.

7. Izquierdo I, Fin C, Schmitz PK, Da Silva RC, Jerusalinsky D, Quillfeldt JA, et al. Memory enhancement by intrahippocampal, intraamygdala, or intraentorhinal infusion of platelet-activating factor measured in an inhibitory avoidance task. Proc Natl Acad Sci US A. 1995; 92(11):5047-51.

8. Gahete MD, Córdoba-Chacón J, Kineman RD, Luque RM, Castaño JP. Role of ghrelin system in neuroprotection and cognitive functions: implications in Alzheimer's disease. Peptides. 2011; 32(11):2225-8.

9. Zhao Z, Liu H, Xiao K, Yu M, Cui L, Zhu Q, et al. Ghrelin administration enhances neurogenesis but impairs spatial learning and memory in adult mice. Neuroscience. 2014; 257:175-85.

10. Babri S, Amani M, Mohaddes G, Mirzaei F, Mahmoudi F. Effects of intrahippocampal injection of ghrelin on spatial memory in PTZ-induced seizures in male rats. Neuropeptides. 2013; 47(5):355-60.

11. Mohaddes G, Mahmoudi F, Mirzaei F, Babri S. Effect of ghrelin intrahippocampal injection on spatial memory in intact male rats. Pharm Sci. 2011; 17(1):49-56.

12. Nazari-Serenjeh F, Darbandi N, Majidpour S, Moradi P. Ghrelin modulates morphine-nicotine interaction in avoidance memory: Involvement of CA1 nicotinic receptors. Brain Res. 2019; 1720:146315.

13. Fallahi S, Babri S, Farajdokht F, Ghiasi R, Zangbar HS, Karimi $\mathrm{P}$, et al. Neuroprotective effect of ghrelin in methamphetaminetreated male rats. Neurosci Lett. 2019; 707:134304.

14. Goshadrou F, Kermani M, Ronaghi A, Sajjadi S. The effect of ghrelin on MK-801 induced memory impairment in rats. Peptides. 2013; 44:60-5.

15. Goshadrou F, Ronaghi A. Attenuating the effect of Ghrelin on memory storage via bilateral reversible inactivation of the basolateral amygdale. Behav Brain Res. 2012; 232(2):391-4. 16. Shafieifar M, Darbandi N, Nazari-Serenjeh F. Involvement of Median Septum Region in Ghrelin Effects on Morphine-Induced Memory Impairment in Passive Avoidance Learning. J Mazandaran Univ Med Sci. 2019; 29(179):28-39.

17. Zahiri H, Rostampour M, Khakpour B, Rohampour K. The effect of ghrelin and adenosine mono phosphate kinase (AMPK) on the passive avoidance memory in male wistar rats. Neuropeptides. 2019; 73:66-70. 